\title{
Expression profiling of small intestinal neuroendocrine tumors identifies subgroups with clinical relevance, prognostic markers and therapeutic targets
}

\author{
Ellinor Andersson ${ }^{1,4}$, Yvonne Arvidsson ${ }^{1,4}$, Christina Swärd ${ }^{2}$, Tobias Hofving ${ }^{1}$, \\ Bo Wängberg ${ }^{2}$, Erik Kristiansson ${ }^{3}$ and Ola Nilsson ${ }^{1}$ \\ ${ }^{1}$ Sahlgrenska Cancer Center, Department of Pathology, Institute of Biomedicine, Sahlgrenska Academy at the \\ University of Gothenburg, Gothenburg, Sweden; ${ }^{2}$ Department of Surgery, Institute of Clinical Sciences, \\ Sahlgrenska Academy at the University of Gothenburg, Gothenburg, Sweden and ${ }^{3}$ Department of \\ Mathematical Sciences, Chalmers University of Technology, Gothenburg, Sweden
}

\begin{abstract}
We wanted to define the transcriptome of small intestinal neuroendocrine tumors in order to identify clinically relevant subgroups of tumors, prognostic markers and novel targets for treatment. Genome-wide expression profiling was conducted on tumor biopsies from 33 patients with well-differentiated neuroendocrine tumors of the distal ileum and metastatic disease at the time of diagnosis. Unsupervised hierarchical clustering analysis identified three groups of tumors. The largest group, comprising half of the tumors, was characterized by longer patient survival and higher expression of neuroendocrine markers, including SSTR2. Tumors with higher grade (G2/3) or gain of chromosome 14 were associated with shorter patient survival and increased expression of cell cycle-promoting genes. Pathway analysis predicted the prostaglandin E receptor 2 (PTGER2) as the most significantly activated regulator in tumors of higher grade, whereas Forkhead box M1 (FOXM1) was the most significantly activated regulator in tumors with gain of chromosome 14. Druggable genes identified from expression profiles included clinically proven SSTR2 and also novel targets, for example, receptor tyrosine kinases (RET, FGFR1/3, PDGFRB and FLT1), epigenetic regulators, molecular chaperones and signal transduction molecules. Evaluation of candidate drug targets on neuroendocrine tumors cells (GOT1) showed significant inhibition of tumor cell growth after treatment with tyrosine kinase inhibitors or inhibitors of HDAC, HSP90 and AKT. In conclusion, we have defined the transcriptome of small intestinal neuroendocrine tumors and identified novel subgroups with clinical relevance. We found specific gene expression patterns associated with tumor grade and chromosomal alterations. Our data also suggest novel prognostic biomarkers and therapies for these patients.
\end{abstract}

Modern Pathology (2016) 29, 616-629; doi:10.1038/modpathol.2016.48; published online 11 March 2016

Patients with small intestinal neuroendocrine tumors usually have metastatic disease at the time of diagnosis. ${ }^{1}$ The clinical course is indolent, but the prognosis for individual patients is difficult to predict. Age, gender, disease stage, urinary 5-hydroxyindoleacetic acid levels and carcinoid heart

Correspondence: Professor O Nilsson, MD, PhD, Department of Pathology, Sahlgrenska Cancer Center, Institute of Biomedicine, Sahlgrenska Academy at the University of Gothenburg, Box 425, Gothenburg SE-405 30, Sweden.

E-mail: ola.nilsson@gu.se

${ }^{4}$ Shared first authors (contributed equally).

Received 6 October 2015; revised 31 January 2016; accepted 31 January 2016; published online 11 March 2016 disease influence patient survival. ${ }^{1-4}$ Assessment of tumor cell proliferation (WHO grade) may serve as a prognosticator, ${ }^{5}$ but biomarkers that accurately predict the clinical course and response to therapy are needed to optimize patient management. ${ }^{6}$ The palliative treatment of patients has been significantly improved, for example, treatment with long-acting somatostatin analogs. ${ }^{7-9}$ Improved patient survival has been reported from single centers, ${ }^{9,10}$ whereas epidemiological studies based on the National Cancer Institute Surveillance, Epidemiology and End Results (SEER) cancer registry have only shown marginal improvement of survival rates. ${ }^{11,12}$ Novel treatment strategies are therefore needed. Molecular characterization of tumors would facilitate the 
development of novel therapies and help to predict sensitivity to anticancer drugs. ${ }^{13-15}$ Recently, the exome of small intestinal neuroendocrine tumors was analyzed by massive parallel sequencing, but no actionable genomic alterations were identified. ${ }^{16,17}$ Expression profiling represents an alternative strategy to obtain a molecular classification of tumors that would predict patient outcome and response to therapy. ${ }^{18,19}$ In this study, we have extended the molecular characterization of small intestinal neuroendocrine tumors by defining the transcriptome of tumors from patients treated at a single center and with long-term follow-up. Expression profiles from these patients allowed us to identify clinically relevant subgroups of tumors and gene expression associated with tumor grade, cytogenetic alterations and patient survival. Novel targets for therapeutic intervention were also identified and evaluated on neuroendocrine tumors cells in vitro.

\section{Materials and methods}

\section{Patients}

Thirty-seven patients who underwent surgery for small intestinal neuroendocrine tumors (ileal carcinoids) at Sahlgrenska University Hospital, Göteborg, Sweden between 1991 and 2009 were included in the study. Tumors from 33 patients were subjected to expression profiling. These patients have been described in a previous study. ${ }^{20}$ Tumor samples from four additional patients were included in the validation experiments. All patients were diagnosed with neuroendocrine tumors of the distal ileum and had metastatic disease at the time of diagnosis (TNM stage IIIB or IV). All tumors were well differentiated by morphological criteria, including organoid growth pattern, light to moderate cellular atypia and low mitotic counts. Tumors were of enterochromaffin cell type, with strong staining for chromogranin A, synaptophysin and serotonin. Grading of tumors according to WHO 2010 (ref. 5) classified 25 tumors as grade 1, 11 tumors as grade 2 and 1 tumor as grade 3 . The grade 3 tumor was a liver metastasis with well-differentiated morphology, mitotic count of 8.4 per 10 high-power fields, and Ki67 index of $30.4 \%$. The primary tumor of this patient was grade 1 (mitotic count $<0.2$ per 10 high-power fields, Ki67 index $0.9 \%$ ). Mean follow-up time was 73 months (median 59, range 5-302 months). The clinicopathological characteristics of the patients are given in Table 1. We obtained consent from the patients and approval from the Regional Ethical Review Board in Gothenburg, Sweden for the use of clinical material for research purposes.

\section{Comparative Genomic Hybridization Microarray}

Tumors from all the patients and the GOT1 cell line were analyzed with respect to somatic copy number alterations using comparative genomic hybridization and oligonucleotide arrays (Agilent Technologies, Palo Alto, CA, USA) as previously described. ${ }^{20}$

\section{Expression Microarray}

Ten patients from the previously reported cohort of small intestinal neuroendocrine tumors ${ }^{20}$ were excluded because of lack of high-quality mRNA $(n=6)$ or poor quality of array hybridization experiments $(n=4)$. Tumor biopsies from the remaining 33 patients (10 primary tumors, 2 lymph node metastases and 21 liver metastases) were included in the study. The purity of tumor biopsies was assessed by light microscopy using hematoxylin and eosin-stained sections and was shown to be in the $60-90 \%$ range. Biopsies of normal small intestinal mucosa from 10 patients who underwent surgery for colorectal carcinomas were used as controls. Control biopsies were confirmed to contain normal small intestinal mucosa by light microscopy (Supplementary Figure S1). The GOT1 cells line was included for comparison, and was cultured as described below. RNA was isolated from fresh-frozen specimens and cell cultures using the miRNeasy Mini Kit (Qiagen) according to the manufacturer's protocol. The quality of extracted RNA was examined by visual inspection after gel electrophoresis using Agilent 2100 Bioanalyzer (Agilent Technologies). Extracts with degraded RNA were excluded. cDNA synthesis, labeling and hybridization were performed according to the One-Color Microarray-Based Gene Expression Analysis protocol v5.7 (Agilent Technologies). Labeled samples were hybridized to $4 \times 44 \mathrm{~K}$ Whole Human Genome Microarrays (G4112F, design ID: 014850, Agilent Technologies). Arrays were scanned using the Agilent G2565BA Microarray Scanner (Agilent Technologies). Images were read and processed using Feature extraction v10.7.1.1 (Agilent Technologies). Expression data are available according to the MIAME standard at the Gene Express Omnibus database (http://www.ncbi.nlm.nih. gov/geo/) with accession number GSE65286.

\section{Statistical Analysis}

The expression microarrays were analyzed using $\mathrm{R}$ software v2.13.0 (www.r-project.org) and the LIMMA package. ${ }^{21}$ The microarrays were background-corrected using the normexp method and normalized using the quantile-quantile method..$^{22,23}$ Differentially expressed genes were identified using linear models and ranked according to the moderated t-statistic. $P$-value adjustment for multiple testing was done using Benjamini-Hochberg false discovery rate. ${ }^{24}$ Cluster analysis of gene expression profiles was done using hierarchical clustering with complete linkage and the Euclidean distance metric. Survival analysis was done using Kaplan-Meier analysis in combination with log-rank tests or using the Cox proportional hazards model, adjusting for gender and age. For the Cox model, significant 
Table 1 Clinicopathological characteristics for 37 patients with small intestinal neuroendocrine tumors

\begin{tabular}{|c|c|c|c|c|c|c|c|c|c|c|c|c|}
\hline $\begin{array}{l}\text { Case } \\
\text { number } d\end{array}$ & $\begin{array}{l}\text { Age at } \\
\text { diagnosis }\end{array}$ & s Gender & $\begin{array}{l}\text { Grade } \\
\text { (WHO } \\
2010 \text { ) }\end{array}$ & $\begin{array}{l}\text { Mitotic } \\
\text { count (per } \\
10 \mathrm{HPF})\end{array}$ & $\begin{array}{l}\text { Ki67 } \\
(\%)\end{array}$ & $\begin{array}{c}\text { Stage } \\
\text { (TNM } \\
\text { 7th ed.) }\end{array}$ & $\begin{array}{c}\text { Follow- } \\
\text { up } \\
\text { (months) }\end{array}$ & $\begin{array}{l}\text { Current } \\
\text { status }\end{array}$ & $\begin{array}{l}\text { Somatostatin } \\
\text { receptor } \\
\text { scintigraphy }\end{array}$ & $\begin{array}{l}\text { Somatostatin } \\
\text { analog } \\
\text { treatment }\end{array}$ & $\begin{array}{l}\text { Primary } \\
\text { surgery }\end{array}$ & $\begin{array}{l}\text { Tumor site } \\
\text { for } \\
\text { experiments }\end{array}$ \\
\hline 1 & 59 & M & G1 & 1.4 & 2.0 & IIIB & 70 & NED & ND & No & Yes & $\mathrm{P}$ \\
\hline 2 & 58 & $\mathrm{~F}$ & G1 & 0.2 & 0.7 & IV & 132 & AWD & Positive & Yes & Yes & $\mathrm{P}$ \\
\hline 3 & 74 & $\mathrm{~F}$ & G1 & $<0.2$ & 0.5 & IV & 83 & DWD & Positive & Yes & Yes & $\mathrm{P}$ \\
\hline 4 & 63 & M & G1 & $<0.2$ & 0.6 & IV & 123 & AWD & Positive & Yes & Yes & $\mathrm{P}$ \\
\hline 5 & 49 & $\mathrm{M}$ & G2 & 1.4 & 6.0 & IV & 5 & DWD & Positive & Yes & Yes & $\mathrm{P}$ \\
\hline 6 & 64 & $\mathrm{~F}$ & G1 & 0.4 & 0.5 & IV & 53 & DWD & Positive & Yes & Yes & $\mathrm{P}$ \\
\hline 7 & 42 & $\mathrm{~F}$ & G1 & 0.6 & 1.9 & IV & 164 & AWD & Positive & Yes & Yes & $\mathrm{P}$ \\
\hline 8 & 81 & $\mathrm{~F}$ & G1 & 0.2 & 2.0 & IV & 35 & DWD & Positive & No & Yes & $\mathrm{P}$ \\
\hline 9 & 68 & $\mathrm{M}$ & G2 & 0.2 & 5.3 & IV & 18 & DWD & ND & Yes & Yes & $\mathrm{P}$ \\
\hline 10 & 79 & $\mathrm{M}$ & G1 & 0.2 & 0.7 & IV & 31 & DWD & Positive & Yes & Yes & $\mathrm{P}$ \\
\hline 11 & 53 & $\mathrm{~F}$ & G1 & $<0.2$ & 0.4 & IV & 141 & AWD & Positive & Yes & Yes & $\mathrm{LN}$ \\
\hline 12 & 59 & $\mathrm{M}$ & G1 & 0.2 & 0.6 & IV & 101 & DWD & Positive & Yes & Yes & LN \\
\hline 13 & 73 & M & G2 & 5.5 & 8.6 & IV & 6 & DWD & Positive & Yes & Yes & $\mathrm{L}$ \\
\hline 14 & 56 & $\mathrm{~F}$ & G3 & 8.4 & 30.4 & IV & 14 & DWD & Positive & Yes & Yes & $\mathrm{L}$ \\
\hline 15 & 53 & $\mathrm{M}$ & G1 & 0.4 & 1.0 & IV & 63 & DWD & Positive & Yes & Yes & $\mathrm{L}$ \\
\hline 16 & 80 & $\mathrm{~F}$ & G1 & 0.4 & 1.1 & IV & 146 & DWD & Positive & Yes & Yes & $\mathrm{L}$ \\
\hline 17 & 71 & $\mathrm{M}$ & G2 & 2.2 & 1.6 & IV & 129 & DWD & ND & Yes & Yes & $\mathrm{L}$ \\
\hline 18 & 56 & $\mathrm{~F}$ & G1 & 0.2 & 1.3 & IV & 49 & DWD & Positive & Yes & Yes & $\mathrm{L}$ \\
\hline 19 & 70 & M & G1 & $<0.2$ & 0.6 & IV & 59 & AWD & Positive & Yes & Yes & $\mathrm{L}$ \\
\hline 20 & 72 & $\mathrm{M}$ & G1 & $<0.2$ & 0.8 & IV & 101 & AWD & Positive & Yes & Yes & $\mathrm{L}$ \\
\hline 21 & 64 & $\mathrm{M}$ & G1 & 0.6 & 0.2 & IV & 97 & DWD & Positive & Yes & Yes & $\mathrm{L}$ \\
\hline 22 & 64 & $\mathrm{~F}$ & G1 & 0.4 & 0.7 & IV & 78 & NED & Positive & Yes & Yes & $\mathrm{L}$ \\
\hline 23 & 61 & $\mathrm{~F}$ & G2 & 4.4 & 16.7 & IV & 15 & DWD & Negative & Yes & Yes & $\mathrm{L}$ \\
\hline 24 & 71 & M & G1 & $<0.2$ & 2.1 & IV & 86 & DWD & Positive & No & Yes & $\mathrm{L}$ \\
\hline 25 & 61 & $\mathrm{~F}$ & G1 & $<0.2$ & 0.7 & IV & 62 & AWD & Positive & Yes & Yes & $\mathrm{L}$ \\
\hline 26 & 70 & $\mathrm{~F}$ & G2 & 7.0 & 11.7 & IV & 14 & DWD & Positive & Yes & Yes & $\mathrm{L}$ \\
\hline 27 & 75 & $\mathrm{~F}$ & G2 & 0.6 & 9.9 & IV & 34 & DWD & Positive & Yes & Yes & $\mathrm{L}$ \\
\hline 28 & 51 & $\mathrm{M}$ & G1 & $<0.2$ & 0.9 & IV & 98 & AWD & Positive & Yes & Yes & $\mathrm{L}$ \\
\hline 29 & 47 & $\mathrm{~F}$ & G2 & 0.6 & 5.5 & IIIB & 302 & AWD & Positive & Yes & Yes & $\mathrm{L}$ \\
\hline 30 & 75 & $\mathrm{~F}$ & G1 & 0.4 & 2.4 & IV & 40 & DWD & Positive & Yes & Yes & $\mathrm{L}$ \\
\hline 31 & 53 & $\mathrm{M}$ & G1 & 0.4 & 2.6 & IIIB & 154 & AWD & Positive & No & Yes & $\bar{L}$ \\
\hline 32 & 74 & M & G2 & 2.2 & 4.7 & IV & 16 & DWD & Positive & Yes & Yes & $\mathrm{L}$ \\
\hline 33 & 74 & $\mathrm{M}$ & G1 & 0.2 & 0.3 & IV & 38 & DWD & Positive & Yes & Yes & $\mathrm{L}$ \\
\hline 34 & 77 & $\mathrm{M}$ & G1 & 0.2 & 1.7 & IV & 27 & DWD & Positive & Yes & Yes & $\mathrm{P}$ \\
\hline 35 & 58 & $\mathrm{M}$ & G2 & 3.0 & 8.7 & IV & 40 & AWD & Negative & Yes & Yes & $\mathrm{P}$ \\
\hline 36 & 55 & $\mathrm{~F}$ & $\mathrm{G} 2$ & 4.4 & 9.6 & IV & 28 & AWD & Positive & Yes & Yes & $\mathrm{LN}$ \\
\hline 37 & 72 & $\mathrm{~F}$ & G1 & 0.2 & 1.4 & IV & 48 & DWD & ND & Yes & Yes & $\mathrm{L}$ \\
\hline
\end{tabular}

Abbreviations: AWD, alive with disease; DWD, dead with disease; F, female; HPF, high-power field; M, male; ND, not determined; NED, no evidence of disease; L, liver metastases; LN, lymph node metastases; P, primary tumor.

parameters were statistically assessed using Wald tests. Predicted drug targets, upstream regulators, canonical pathways and biological functions were identified using QIAGEN Ingenuity Pathway Analysis (IPA ${ }^{\circledR}$, QIAGEN; www.qiagen.com/ingenuity). ${ }^{25}$ Student's $t$-test was used to assess significance of differences in gene expression measured by qPCR (validation experiments).

\section{Quantitative Real-Time PCR (qPCR)}

RNA extraction from tumor tissue was performed as described above. cDNA was synthesized using the High Capacity RNA-to-cDNA Kit (Life Technologies). mRNA expression levels were analyzed using predesigned TaqMan Gene Expression Assay (Life Technologies): ARHGAP11A (Hs00207575_m1), ASPM (Hs00411505_m1), BIRC5 (Hs04194392_s1), CALB1
(Hs01077197_m1), CDCA5 (Hs00293564_m1), CDKN2B (Hs00793225_m1), CENPF (Hs01118845_m1), CTTNBP2 (Hs00364312_m1), FOXM1 (Hs01073586_m1), GAPDH (Hs99999905 m1), GPR110 (Hs00228100 m1), GTSE1 (Hs00212681_m1), HPRT1 (Hs02800695_m1), KIF18B (Hs00977732_m1), POC1A (Hs00248813_m1) and PTTG1 (Hs00851754_u1). The PCR reactions were performed using a 7500 Fast Real-Time PCR System (Applied Biosystems). The samples were analyzed in duplicate. The levels of mRNA expression are reported relative to those of the two housekeeping genes GAPDH and HPRT1.

\section{Immunohistochemistry}

Formalin-fixed, paraffin-embedded tumor material was studied by immunohistochemistry. Sections $(3-4 \mu \mathrm{m})$ were placed on positively charged glass slides and 


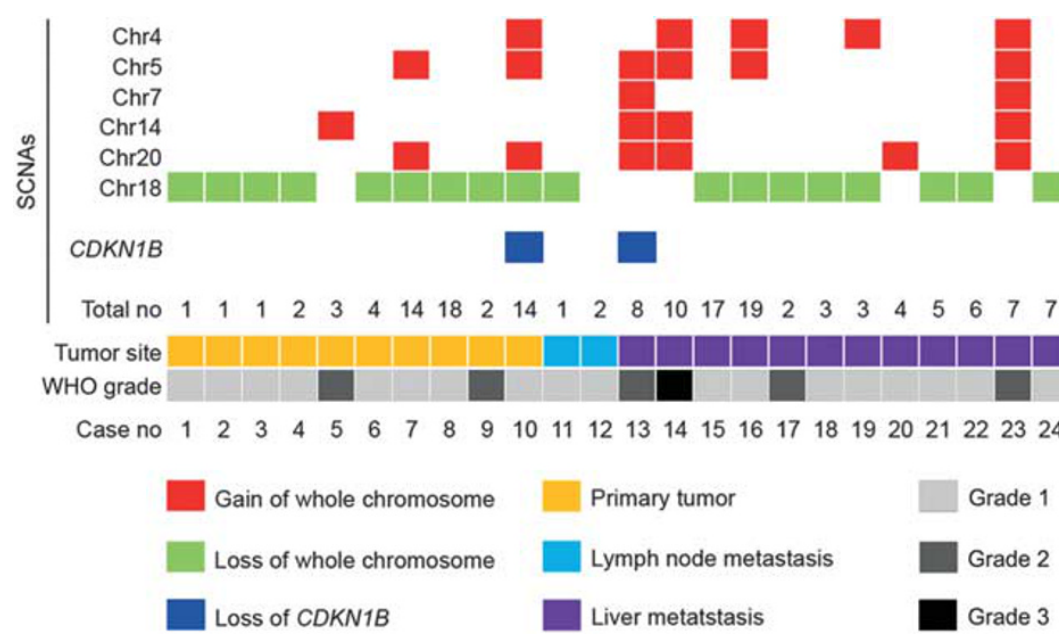

Figure 1 Somatic copy number alterations in small intestinal neuroendocrine tumors. Loss of chromosome 18 was the most frequent somatic copy number alteration (SCNA). Gains were frequently detected on chromosomes 4, 5, 7, 14 and 20. Loss of CDKN1B, which is frequently mutated in small intestinal neuroendocrine tumors, was observed in three tumors. The average number of somatic copy number alterations per tumor was 8.0 (range 1-21). Tumor site and WHO grade for each tumor is indicated. Details on comparative genomic hybridization profiles of case 1-33 have been published elsewhere (Andersson et al. ${ }^{20}$ ). Somatic copy number alterations in the GOT1 cell line are shown for comparison. GOT1 cells had 21 losses, including loss on chromosome 18 (for details see Supplementary Table S1).

treated in Dako PT-Link using EnVision ${ }^{\text {TM }}$ FLEX Target Retrieval Solution (high $\mathrm{pH}$ ). Immunohistochemical staining was performed in a Dako Autostainer Link using EnVision ${ }^{\mathrm{TM}}$ FLEX with EnVision ${ }^{\mathrm{TM}}$ FLEX+ (LINKER) according to the manufacturer's instructions (DakoCytomation). Positive and negative controls were included in each run. The following primary antibodies were used: anti-ARHGAP11A (HPA040419, Sigma Aldrich), anti-ASPM (09-066, Merck Millipore), anti-BIRC5 (sc-17779, Santa Cruz Biotechnology), anti-CALB1 (10R-C106A, Fitzgerald), anti-CDCA5 (HPA023691), anti-CDKN2B (MA1-12294, Thermo Fisher Scientific), anti-CENPF (ab90, Abcam), antiCTTNBP2 (17893-1-AP, Proteintech), anti-FOXM1 (sc-271746, Santa Cruz Biotechnology), anti-GPR110 (LS-A2019, LifeSpan Biosciences), anti-GTSE1 (213191-AP, Proteintech), anti-KIF18B (ab121798, Abcam), anti-POC1A (HPA040600, Sigma Aldrich) and antiPTTG1 (ab3305, Abcam). Stained sections were evaluated using light microscopy. For cytoplasmic and/or membranous labeling, sections were evaluated visually and tumors considered positive if $>10 \%$ of tumor cells were labeled. For nuclear labeling, manual counting was performed on printed images and the percentage of labeled tumor cell nuclei calculated.

\section{In Vitro Experiments with GOT1 Cells}

The GOT1 cell line, derived from a patient with metastatic small intestinal neuroendocrine tumor, was maintained under culture conditions previously described. ${ }^{26}$ These cells were treated with $0-10 \mu \mathrm{M}$ cabozantinib, MK-2206, P276-00, regorafenib, sorafenib, sunitinib, veliparib, vorinostat or $0-5 \mu \mathrm{M}$ alvespimycin (Selleck Chemicals) for 4 days. All drugs were dissolved in DMSO. The viability of GOT1 cells was measured using AlamarBlue ${ }^{\circledR}$ (Molecular Probes) according to the manufacturer's instructions. Three experiments were performed independently in triplicate.

\section{Results}

\section{Somatic Copy Number Alterations in Small Intestinal Neuroendocrine Tumors}

All small intestinal neuroendocrine tumors had somatic copy number alterations, ranging from 1 to 21 per tumor. The average number of alterations per tumor was 8.0, with losses being more common than gains. The most frequent alteration was loss of chromosome 18, which was observed in 24 of 37 tumors $(65 \%)$. The most frequent gains occurred on chromosomes 4, 5, 7, 14 and 20, affecting 19 out of 37 tumors $(51 \%)$. Loss of $C D K N 1 B$ was observed in three tumors (8\%). Grade 1 tumors frequent had loss of chromosome $18(21 / 25)$, whereas grade 2 tumors frequently had gain of chromosome 14 (7/11). The grade 3 tumor also had gain of chromosome 14, as well as gains on chromosomes 4, 5 and 20 . A summary of frequent somatic copy number alterations in tumor biopsies is given in Figure 1. The GOT1 cell line had 21 copy number alterations, all of which were losses, including loss on chromosome 18. GOT1 cells had no loss of $C D K N 1 B$. Full information on alterations in GOT1 cells is given in Supplementary Table S1. 


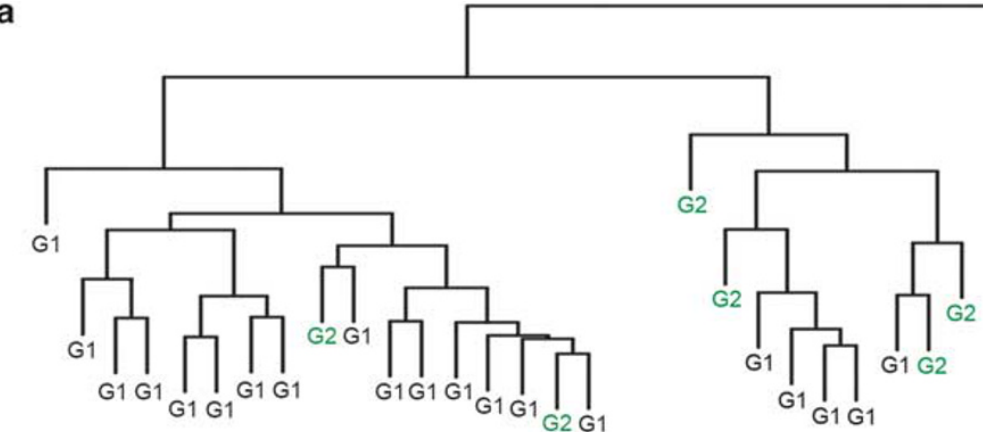

Cluster A

Cluster B

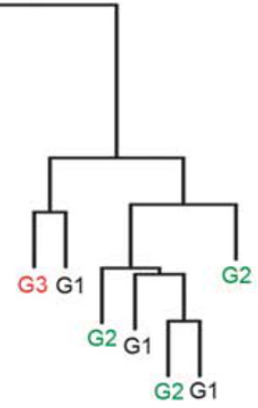

Cluster C b

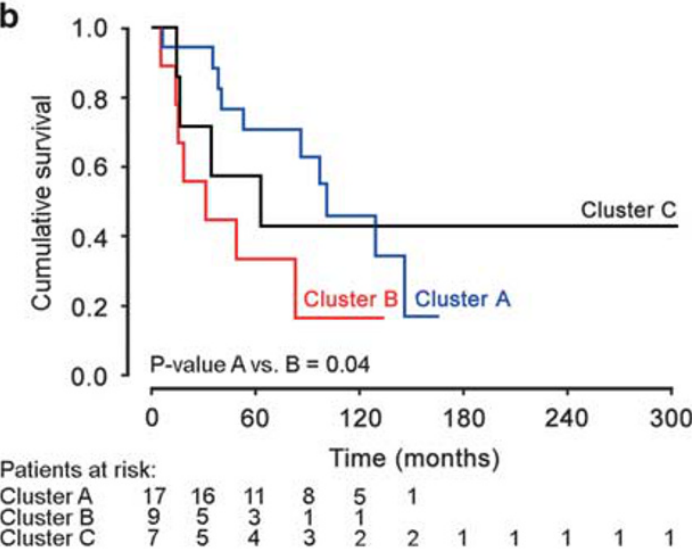

d

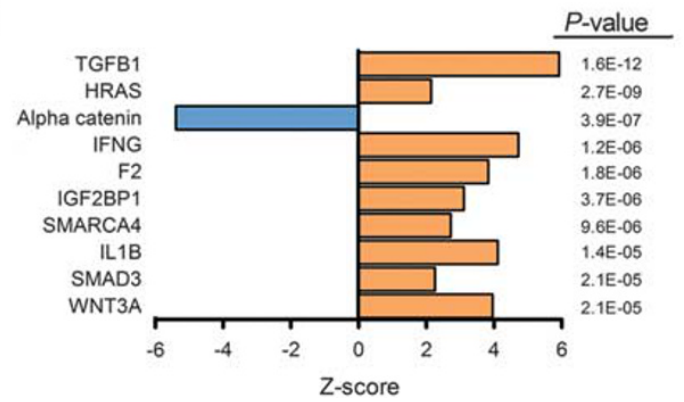

C

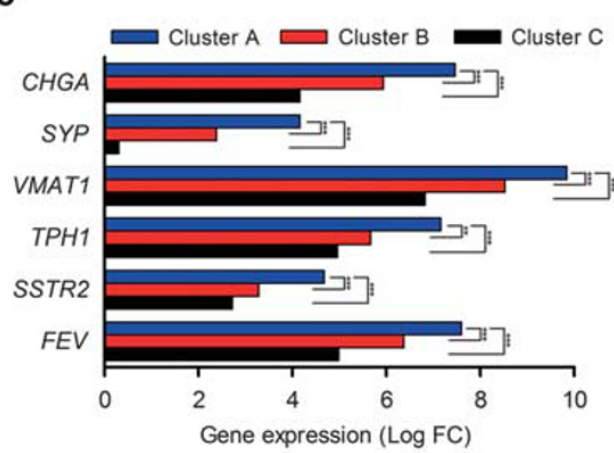

e

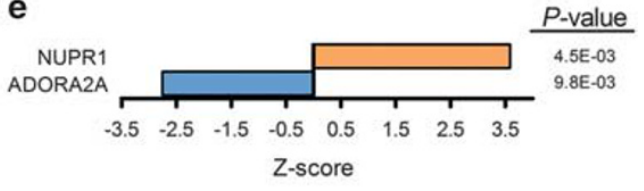

Figure 2 Clustering analysis of small intestinal neuroendocrine tumors. (a) Unsupervised hierarchical clustering analysis of gene expression profiles. Three distinct tumor clusters were identified, denoted clusters A, B and C. Tumor grade according to WHO 2010 is indicated: G1, grade 1; G2, grade 2; G3, grade 3. (b) Survival analysis of tumor clusters using Kaplan-Meier analysis. Shorter overall survival was observed for patients in clusters B $(P<0.05)$ compared with patients in cluster A. (c) Expression of marker genes for small intestinal neuroendocrine tumors in clusters A, B and C. Highest expression of marker genes was observed in cluster A followed by cluster B and cluster C. Significance is shown relative to cluster A. ${ }^{*} P$-value $<0.01,{ }^{*} *{ }^{*} P$-value $<0.001$. (d) Pathway analysis predicted 56 upstream regulators significantly activated (Z-score $\geq 2$ ) or inhibited (Z-score $\leq 2$ ) in cluster B vs cluster A (overlapping $P$-value $<0.01$ ). Shown are the 10 upstream regulators with the highest overlapping $P$-value. Of these, TGFB1 was the most activated (Z-score 5.9) and alpha catenin was the most inhibited (Z-score - 5.4). (e) Pathway analysis of cluster C vs cluster A identified two upstream regulators: NUPR1 (activated in C) and ADORA2A (inhibited in C). Upstream regulators were obtained by pathway analysis (IPA) using the 2000 most significantly differentially expressed genes between the clusters.

\section{Expression Profiling Identified Three Clusters of Small Intestinal Neuroendocrine Tumors with Different Patient Survival}

First, we compared the global gene expression profiles of tumors with that of normal small intestinal mucosa. Marker genes for small intestinal neuroendocrine tumors were highly upregulated in all tumor biopsies, confirming the authenticity of the tumor material (Figure 2c). Second, we analyzed the expression profiles of tumors (excluding small intestinal mucosa) by unsupervised hierarchical clustering analysis and found three separate tumor clusters (Figure 2a). The largest cluster (cluster A) contained more than half of the tumors (17/33), whereas the two smaller clusters (clusters B and C) each contained approximately one-quarter of the tumors (9/33 and 7/33). Tumors in cluster A were predominantly of lower grade (G1), whereas tumors in clusters $\mathrm{B}$ and $\mathrm{C}$ were frequently of higher grade 
Table 2 Clinicopathological characteristics for small intestinal neuroendocrine tumor clusters

\begin{tabular}{|c|c|c|c|c|}
\hline & All tumors & Cluster A & Cluster B & Cluster $C$ \\
\hline Number of cases & 33 & 17 & 9 & 7 \\
\hline \multicolumn{5}{|l|}{ Gender } \\
\hline Female & 16 & 8 & 5 & 3 \\
\hline Male & 17 & 9 & 4 & 4 \\
\hline Mean age (years) & 64.2 & 65.6 & 63.8 & 61.4 \\
\hline \multicolumn{5}{|l|}{ Grade (WHO 2010) } \\
\hline G1 & 23 & 15 & 5 & 3 \\
\hline G2 & 9 & 2 & 4 & 3 \\
\hline G3 & 1 & 0 & 0 & 1 \\
\hline \multicolumn{5}{|l|}{ Stage (TNM 7th ed.) } \\
\hline Stage IIIB & 3 & 0 & 1 & 2 \\
\hline Stage IV & 30 & 17 & 8 & 5 \\
\hline $\begin{array}{l}\text { Mean follow-up } \\
\text { (months) }\end{array}$ & 77.5 & 85.6 & 46.3 & 97.7 \\
\hline \multicolumn{5}{|l|}{ Site of biopsy } \\
\hline Primary tumors & 10 & 4 & 6 & 0 \\
\hline Metastases & 23 & 13 & 3 & 7 \\
\hline \multicolumn{5}{|c|}{ Somatic copy number alterations } \\
\hline $\begin{array}{l}\text { Gain of } \\
\text { chromosome } 14\end{array}$ & 7 & 1 & 3 & 3 \\
\hline $\begin{array}{l}\text { No gain of } \\
\text { chromosome } 14\end{array}$ & 26 & 16 & 6 & 4 \\
\hline
\end{tabular}

(G2/3). A higher proportion of tumors in clusters B and $\mathrm{C}$ had gain of chromosome 14. Clusters A and B contained both primary and metastatic tumors, whereas cluster C contained metastases only. The clinical characteristics of patients in the three tumor clusters were similar with respect to age, gender and tumor stage (Table 2, Supplementary Figure S2). Patient survival, however, was significantly shorter $(P<0.05)$ for patients in cluster B than for patients in cluster A (Figure 2b).

\section{Gene Expression Profiles of Tumor Clusters}

We analyzed the global gene expression profiles of tumor clusters by comparing the gene expression of each cluster with that of normal small intestinal mucosa. The most upregulated genes in clusters A and B contained a high proportion of genes related to neurosecretory function and formation of cellular protrusions, whereas the most upregulated genes in cluster C contained a high proportion of genes associated with angiogenesis and cytoskeletal organization (Supplementary Tables S2-4). IPA predicted REST (inhibited), PHF21A (activated) and NEUROD1 (activated) as upstream regulators in clusters A and B, whereas FGF2 (activated) was the most significant upstream regulator in cluster C (Supplementary Table S5). REST, PHF21A and NEUROD1 regulate neuronal and enteroendocrine cell differentiation. We therefore compared tumor clusters with respect to neuroendocrine marker genes and found the highest expression of these genes in cluster A, followed by cluster B and cluster C (Figure 2c). Next, we analyzed the gene expression profiles of tumor clusters (excluding small intestinal mucosa) by performing a pairwise comparison of clusters. Comparison of cluster A and cluster B predicted TGFB1 and HRAS as the most significantly activated upstream regulators in cluster B (Figure 2d). Comparison of cluster A and cluster $\mathrm{C}$ predicted NUPR1 as the most significantly activated upstream regulator in cluster C (Figure 2e).

Tumor Grade is Associated with Altered Expression of Genes Related to Proliferation and Microenvironment

In order to obtain information on the molecular alterations associated with tumor grade, we compared the expression profiles of grade 1 tumors with those of grade $2 / 3$ tumors. A total of 70 genes were differentially expressed between the two groups (adjusted $P$-value $<0.05)$. A substantial proportion of the genes were involved in different steps of tumor cell replication, whereas other genes were related to the interaction between tumor cells and their microenvironment (Figure 3a). Pathway analysis predicted activation of several upstream regulators in grade $2 / 3$ tumors, which promote cell cycling. The most significant upstream regulator, however, was prostaglandin E receptor 2 (PTGER2), which mediates proinflammatory signals and stimulates tumor growth and invasion (Figure 3b). Pathway analysis was used to generate a regulatory network including CCND1, CSF2, Vegf and HGF, which together explained the growth-promoting effects of differentially expressed genes in grade $2 / 3$ tumors (Figure 3c). Patient survival was significantly longer for grade 1 tumors. These tumors showed a higher degree of neuroendocrine differentiation, as indicated by higher expression of marker genes (Figures 3d and e).

\section{FOXM1 Expression is Upregulated in Small Intestinal Neuroendocrine Tumors Carrying Gain of Chromosome 14}

We have previously shown that small intestinal neuroendocrine tumors carrying chromosomal gains, notably gain of chromosome 14, have worse prognosis (Supplementary Figure S3). ${ }^{20}$ To extend the molecular characterization of these tumors, we compared the expression profiles of tumors with gain of chromosome 14 to those of tumors with no gain of chromosome 14. Altogether, 181 genes were differentially regulated between the two tumor groups (adjusted $P$-value $<0.05$ ). Regulated genes were distributed over all chromosomes, with only two significantly upregulated genes located on chromosome 14 (HECTD1 and STYX). Differentially expressed genes were involved in cell cycle progression, mitotic spindle formation, apoptosis, DNA 
a

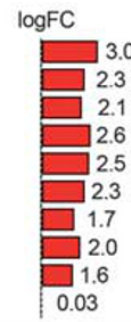

Adj. $P$-value
$1.1 \mathrm{E}-02$
$1.1 \mathrm{E}-02$
$1.1 \mathrm{E}-02$
$1.2 \mathrm{E}-02$
$1.6 \mathrm{E}-02$
$2.0 \mathrm{E}-02$
$3.3 \mathrm{E}-02$
3.3E-02
$3.3 \mathrm{E}-02$
$3.3 \mathrm{E}-02$
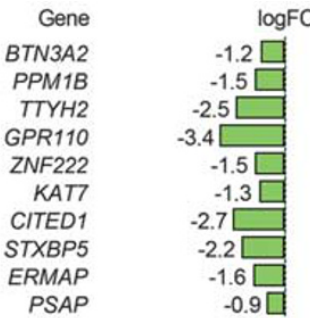

$\log \mathrm{FC}$

Adj. $P$-value

BIRC5

FAM64A

TYMS

KIF2OA

GABRA2

3.3E-02

Biological function
cell cycle progression

cell cycle progression

liver cancer

maturation of hematopoietic progenitor cells

endocytosis

growth of digestive organ tumor

b

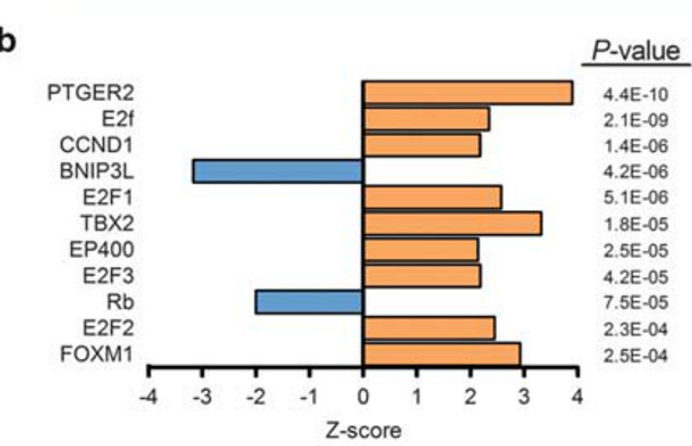

d

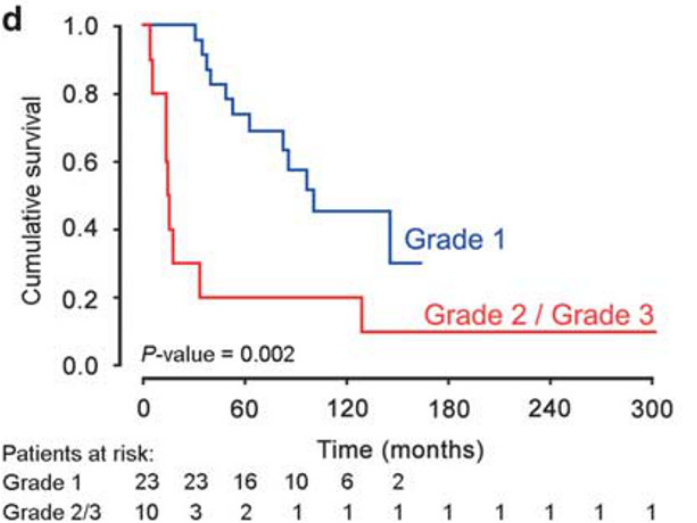

\begin{tabular}{lll}
$P$-value & Z-score & No. of molecules \\
\hline $1.3 \mathrm{E}-05$ & 2.38 & 67 \\
$3.8 \mathrm{E}-04$ & 2.41 & 220 \\
$1.9 \mathrm{E}-03$ & -2.14 & 8 \\
$2.6 \mathrm{E}-03$ & -2.74 & 22 \\
$6.2 \mathrm{E}-03$ & 2.62 & 8
\end{tabular}

1.1. -02

1.3E-02

3.3E-02

3.3E-02

3.7E-02

$3.8 \mathrm{E}-02$

$3.9 \mathrm{E}-02$

4.2E-02

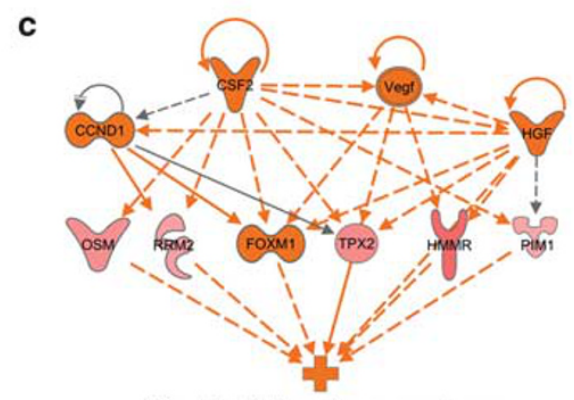

Growth of digestive organ tumor

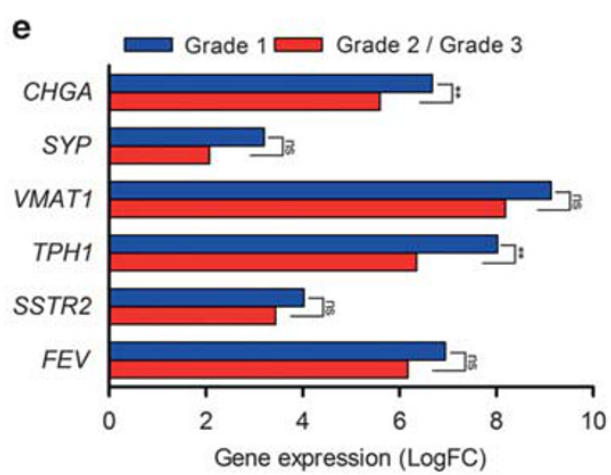

Figure 3 Gene expression profiles associated with tumor grade in small intestinal neuroendocrine tumors. (a) Gene expression profiles of grade 2/3 tumors were compared with those of grade 1 tumors. Differentially regulated genes were related to cell cycle progression (BIRC5, FAM64A, CDK1 and PPM1B), mitotic spindle formation and cytokinesis (NUSAP1, ASPM, TROAP and KIF20A), apoptosis (BIRC5) and DNA repair (NUSAP1 and TYMS). Other altered functions included cell mobility and metastasis (HMMR, TROAP and PSAP), immune response (BTN3A2), and neuroendocrine function (GABRA2, STXBP5 and PSAP). The 10 most upregulated genes (left) and downregulated genes (right) in grade $2 / 3$ tumors have been sorted by increasing adjusted $P$-values. $\log$ FC, $\log$ fold change. Pathway analysis (IPA) found 'cell cycle progression' and 'liver cancer' to be the most significantly activated biological functions in grade $2 / 3$ tumors. (b) The most significantly activated upstream regulator in grade 2/3 tumors was PTGER2, a prostanoid receptor mediating proinflammatory signals. A number of upstream regulators controlling cell cycle progression were also activated, including E2F1, E2F2, E2F3, CCND1, TBX2 and FOXM1. On the other hand, the inducer of apoptosis BNIP3L and the tumor-suppressor Rb were inactivated. Listed upstream regulators are sorted by overlapping $P$-value $<0.01$ with a Z-score $\leq-2$ or $\geq 2$. (c) Stimulation of tumor growth by differentially expressed genes in grade $2 / 3$ tumors. Pathway analysis (IPA) predicted a regulatory network consisting of four upstream regulators, CCND1, CSF2, VEGF and HGF, whose conserted action on five target genes, OSM, RRM2, FOXM1, TPX2, HMMR and PIM1, explained the higher proliferation rate in tumors of grade 2/3. Arrows indicate stimulation via direct mechanisms (solid line) or indirect mechanisms (dotted line). Orange symbols indicate upregulated genes in biopsies from tumors of grade 2/3. (d) Survival analysis of tumors with respect to WHO grade using Kaplan-Meier analysis. Shorter overall survival was observed for patients with grade 2/3 tumors than for those with grade 1 tumors $(P<0.005)$. (e) Expression of marker genes for small intestinal neuroendocrine tumors in grade 1 and grade 2/3 tumors. Highest expression of marker genes was observed in grade 1 tumors. Differential expression of $C H G A$ and $T H P 1$ reached statistical significance. ${ }^{*} P$-value $<0.01$; NS, not significant. 
repair, cell mobility and neuroendocrine function (Figure 4a). Pathway analysis identified Forkhead box M1 (FOXM1), a transcription factor controlling cell cycle progression and response to DNA damage, as the most significant upstream regulator activated in tumors with gain of chromosome 14 (Figure 4b). Differential expression of FOXM1 was confirmed by qPCR and increased FOXM1 labeling index was shown by immunohistochemistry (Figure 4c). a

\begin{tabular}{|c|c|c|}
\hline Gene & $\log \mathrm{FC}$ & Adj. $P$-value \\
\hline ARL14 & 3.9 & $3.5 \mathrm{E}-03$ \\
\hline TMEM184A & 1.6 & $1.6 \mathrm{E}-02$ \\
\hline FOXM1* & 2.2 & $1.8 \mathrm{E}-02$ \\
\hline POC1A* & ] 1.8 & $1.8 \mathrm{E}-02$ \\
\hline HMGB3P1 & 1.7 & $1.8 \mathrm{E}-02$ \\
\hline HECTD1 & 0.8 & $1.8 \mathrm{E}-02$ \\
\hline ASPM* & 2.5 & $1.9 \mathrm{E}-02$ \\
\hline SPATA25 & 1.3 & $1.9 \mathrm{E}-02$ \\
\hline$B I R C 5^{*}$ & $\square 2.8$ & $2.3 \mathrm{E}-02$ \\
\hline CEP164 & ] 1.4 & 2.3E-02 \\
\hline
\end{tabular}

Gene
PPP2R2B
FAR2
MBD3L2
CNGA3
CTTNBP2*
TMEM64
KIF16B
RESP18
CALB1*
BBX

Adj. $P$-value
$3.5 \mathrm{E}-03$
$3.5 \mathrm{E}-03$
$3.5 \mathrm{E}-03$
$1.4 \mathrm{E}-02$
$1.5 \mathrm{E}-02$
$1.5 \mathrm{E}-02$
$1.6 \mathrm{E}-02$
$1.6 \mathrm{E}-02$
$1.6 \mathrm{E}-02$
$1.7 \mathrm{E}-02$

b

\begin{tabular}{|c|c|c|c|c|}
\hline \multirow{2}{*}{$\begin{array}{l}\text { Upstream } \\
\text { Regulator }\end{array}$} & \multirow[t]{2}{*}{ Z-score } & \multirow{2}{*}{$\begin{array}{l}P \text {-value of } \\
\text { overlap }\end{array}$} & \multicolumn{2}{|l|}{ Target molecules in dataset } \\
\hline & & & Upregulated & Downregulated \\
\hline FOXM1 & 2.67 & 7.3E-05 & $\begin{array}{l}\text { BIRC5, CCNB2, CDC20, CDK1, CDKN3, CENPF, } \\
\text { FOXM1, GTSE1, KIF2OA, PLK4, PRC1 }\end{array}$ & $C D K 2^{*}, C D K N 1 B$ \\
\hline EHF & 2.16 & 4.0E-04 & CDK1, E2F1, ERBB2, TNFRSF10B & CDKN1B \\
\hline TBX2 & 3.16 & 1.7E-03 & $\begin{array}{l}\text { CDC6, CDCA5, CDK1, E2F1, E2F7, EZH2, } \\
\text { FOXM1, MXD3, PKMYT1, PRC1 }\end{array}$ & \\
\hline CDKN2A & -2.06 & 2.1E-03 & $\begin{array}{l}\text { BIRC5, CDCA5, CDCA7L, CDK1, CDKN2B, E2F1, } \\
\text { E2F2, EZH2, GTSE1*, MKI67, SH3BP2* }\end{array}$ & $\begin{array}{l}\text { CDK2*, CDKN1B, } \\
\text { CITED2, FMN2, NR3C1, } \\
\text { POLD3* }{ }^{*} \text { PTK2B, SUZ12* }\end{array}$ \\
\hline HGF & 2.92 & 3.7E-03 & $\begin{array}{l}\text { BIRC5, CDC20, CDC6, CDK1, CDKN2B }{ }^{\star}, C D K N 3 \text {, } \\
\text { CENPF, CLCF1, COL4A1 }{ }^{\star}, F N 1, F O X M 1, H K 2, \\
\text { HMMR, HMOX1, MAP2K3, MKI67, NDC80, } \\
\text { PKMYT1, PLK4, PRC1, PTTG1, TNFAIP3, } \\
\text { TNFRSF10B, TPX2, UBE2C }\end{array}$ & $\begin{array}{l}\text { CDK2*, CDKN1, FGF7, } \\
\text { GRB10*, ITGB3BP*, } \\
\text { KRR1, NCOA2*, NEK3*, } \\
\text { NRIP1 }\end{array}$ \\
\hline Vegf & 2.44 & $6.8 \mathrm{E}-03$ & $\begin{array}{l}\text { APOM, BIRC5, CDC20, CDC6, CDK1, CDKN3, } \\
\text { CENPF, CLCF1, ELN* FOXM1, HK2, HMMR, } \\
\text { HMOX1, MAP2K3, MKI67, NDC80, PKMYT1, } \\
\text { PLK4, PRC1, TNFRSF10B, TPX2, UBE2C }\end{array}$ & 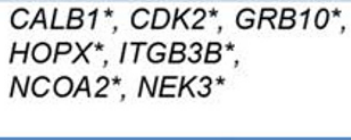 \\
\hline
\end{tabular}

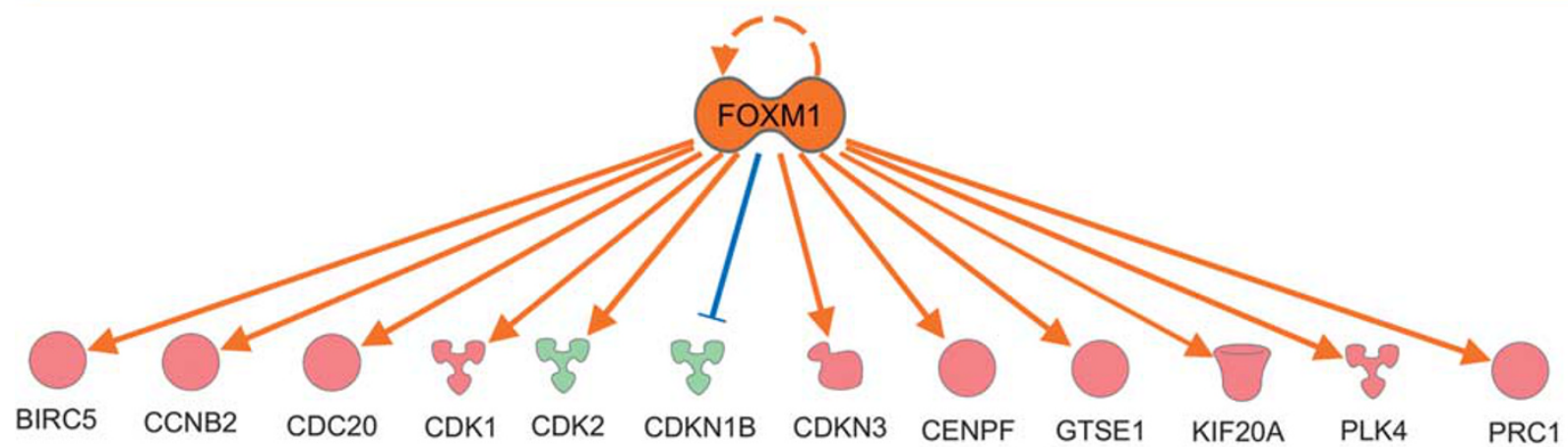

C

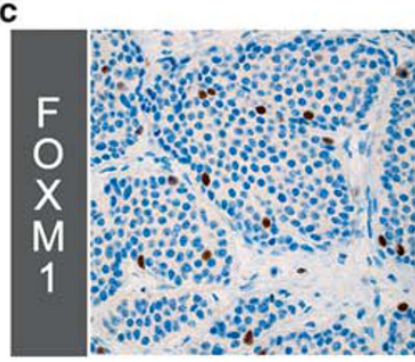

Gain Chr14

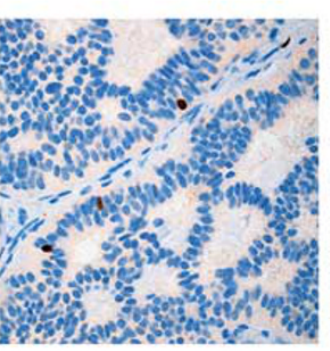

No Gain Chr14
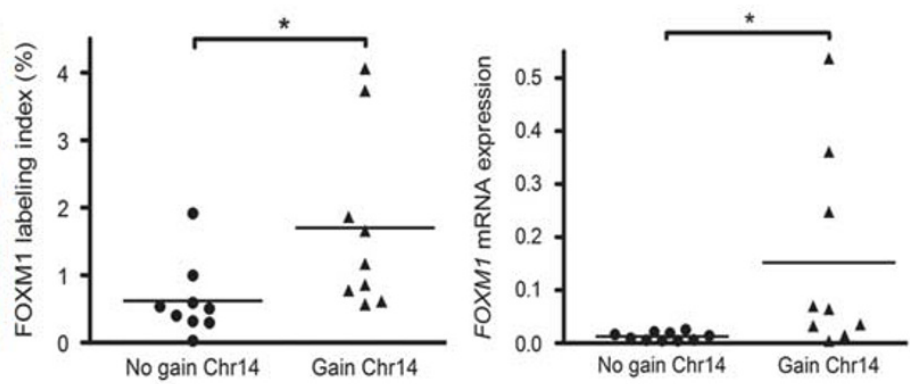

Figure 4 For caption see page 624. 


\section{Identification of Gene Expression Related to Patient Survival in Small Intestinal Neuroendocrine Tumors}

The molecular mechanisms underlying the aggressive behavior of small intestinal neuroendocrine tumors are largely unknown. In order to identify these mechanisms, we compared gene expression values in individual tumor biopsies with patient survival using a Cox proportional hazards model. Analysis of expression profiles from all 33 tumors identified 168 genes associated with patient survival (adjusted $P$-value $<0.12$ ) (Figure 5a). Only two of the genes (BIRC5 and $C D K N 1 B$ ) have previously been reported to be prognostic markers for small intestinal neuroendocrine tumors, ${ }^{27-29}$ whereas 31 genes have been reported to be prognostic markers in other malignancies. In order to identify gene expression that contributes to shorter survival in tumors of cluster B, grade $2 / 3$ and gain of chromosome 14 , we compared survival-related genes with differentially regulated genes in these subgroups (Figure 5b). There was a substantial overlap of survival-related genes with differentially expressed genes in these subgroups (49 of 168), frequently genes related to cell proliferation and apoptosis (Figure 5c).

\section{Prediction of Therapeutic Targets in Small Intestinal Neuroendocrine Tumors}

There have been no known genomic alterations that would predict the therapeutic response in small intestinal neuroendocrine tumors. We therefore searched expression profiles for signaling pathways that may be targeted for therapy. We interrogated the most upregulated genes in tumors relative to small intestinal mucosa for corresponding anticancer drugs that are currently in clinical trials. Analysis including all tumors identified a total of 16 candidate targets, including $\mathrm{G}$ protein-coupled somatostatin receptor 2 (SSTR2), receptor tyrosine kinases (RET, FGFR1/3, PDGFRB and FLT1), the growth factor VEGFB, nuclear kinases (CDK4/9), the transcriptional regulator HDAC5, molecular chaperones (HSP90AA1 and HSP90AB1), the signal transduction molecule AKT3 and the DNA repair enzyme PARP1
(Table 3). When tumor clusters were analyzed separately, we identified a distinct set of candidate targets for each cluster (Supplementary Figures S5; Supplementary Tables S6-8). Seven candidate targets were highly expressed in all three clusters, including SSTR2, which had significantly higher expression in cluster A (Supplementary Figure S6). When grade 1 tumors and grade 2/3 tumors were analyzed separately, the majority of candidate targets were represented in both groups (Supplementary Figure S8; Supplementary Table S9). A selection of predicted anticancer drugs was evaluated on neuroendocrine tumor cells in vitro. We chose the GOT1 cell line as model system because it was derived from a metastatic small intestinal neuroendocrine tumor. The validity of the GOT1 cell line was supported by the presence of copy number alterations typical of small intestinal neuroendocrine tumors (loss on chromosome 18) and high expression of marker genes (CHGA, SYP, VMAT1, TPH1 and SSTR2) (Supplementary Figure S8). Furthermore, we found that GOT1 cells expressed all candidate drug targets identified in tumor biopsies (Table 3). Evaluation of tyrosine kinase inhibitors sorafenib, sunitinib, regorafenib and cabozantinib, which inhibit candidate targets RET, FGFR1, PDGFRB and FLT1, showed significant inhibition of GOT1 cell growth with $\mathrm{IC}_{50}$ values in the micromolar range. Inhibitors of AKT (MK-2206), HDAC (vorinostat), HSP90 (alvespimycin) and CDK4/9 (P276-00) also effectively inhibited GOT1 cell growth, whereas inhibition of PARP1 (veliparib) had no effect (Figure 6).

\section{Discussion}

We have defined the transcriptome of small intestinal neuroendocrine tumors and shown that tumors can be divided into distinct groups based on their gene expression profiles. Major differences in expression profiles were identified between tumor groups, involving a substantial number of signaling pathways. We found that transforming growth factor beta (TGFB1), a master regulator of epithelial-

Figure 4 Gene expression profiles of small intestinal neuroendocrine tumors carrying gain of chromosome 14. (a) Differentially expressed genes in tumors with gain of chromosome 14 were frequently related to cell cycle progression and mitotic spindle formation (FOXM1, POC1A, ASPM, BIRC5, CEP164 and BBX), apoptosis (BIRC5, PPP2R2B and CALB1), DNA damage response (FOXM1, CEP164 and BBX), cell mobility (HECTD1 and CTTNPB2) and neuroendocrine function (CNGA3, CTTNBP2, RESP18 and CALB1). The 10 most upregulated genes (left) and downregulated genes (right) have been sorted by increasing adjusted $P$-values. Asterisks indicate genes localized to tumor cell by immunohistochemistry and confirmed to be differentially expressed by qPCR (Supplementary Figure S4). logFC, log fold change. (b) FOXM1, EHF, TBX2 and CDKN2A were the most significant upstream regulators in tumors with gain of chromosome 14. All of these regulators directly control cell cycle progression and apoptosis, as predicted by pathway analysis (IPA). Other upstream regulators included HGF and VEGF, which promote epithelial-mesenchymal transition and angiogenesis. The upstream regulators listed have been sorted by overlapping $P$-value $<0.01$ with a Z-score $\leq-2$ or $\geq 2$. The effect of FOXM1 on downstream target genes is illustrated. Direct stimulatory effects (arrow) and inhibitory effects (blunted line) of FOXM1 are indicated. The differential expression of target genes in tumor biopsies are indicated by orange symbols (upregulated in tumors with gain of chromosome 14) and green symbols (downregulated in tumors with gain of chromosome 14). (c) Localization of FOXM1 protein in tumor biopsies by immunohistochemistry showed significantly higher nuclear labeling in tumors with gain of chromosome 14 than in tumors without gain of chromosome 14. qPCR analysis confirmed differential expression of FOXM1 mRNA in the two tumor groups. 
mesenchymal transition and metastasis, ${ }^{30,31}$ was activated in a subset of tumors (cluster B), which was associated with shorter patient survival. We also found nuclear protein 1 (NUPR1), a chromatin binding protein regulating stress response and resistance to chemotherapy, ${ }^{32}$ to be activated in another subset of tumors (cluster C). Significantly deregulated pathways in tumor clusters may indicate molecular mechanism involved in the formation of these tumors. The clinical relevance of identified tumor groups was evident, as cluster designation correlated to patient survival independent of tumor grade and stage. The clinical characteristics did not reveal any difference in age, gender or tumor stage between tumor groups. However, the two smaller groups (clusters B and C) contained a higher proportion of tumors with higher grade and chromosomal gains. Tumors in cluster $\mathrm{C}$ were the most heterogeneous with respect to tumor grade, containing the only grade 3 tumor in the study. The reason for this heterogeneity was not evident. However, unsupervised clustering divided tumors into groups based on similarities in their expression profiles. Tumors in cluster C shared a set of deregulated a

\begin{tabular}{|c|c|c|}
\hline Gene & ExpCoef & $P$-value \\
\hline TRIM40 & 13.5 & $5.9 \mathrm{E}-6$ \\
\hline FAM9C & 9.9 & $1.2 \mathrm{E}-5$ \\
\hline GTSE1* & 2.3 & $1.5 \mathrm{E}-5$ \\
\hline PPBPL2 & 4.5 & $1.7 \mathrm{E}-5$ \\
\hline ADAMTS12 & 4.1 & $2.6 \mathrm{E}-5$ \\
\hline$C D C A 5^{\star}$ & 3.3 & $3.2 \mathrm{E}-5$ \\
\hline CENPF* & 4.2 & $3.6 \mathrm{E}-5$ \\
\hline LOXHD1 & 3.4 & $4.0 \mathrm{E}-5$ \\
\hline ARHGAP $11 A^{*}$ & 3.0 & $4.2 \mathrm{E}-5$ \\
\hline $\operatorname{IRX} 6$ & 5.4 & $6.0 \mathrm{E}-5$ \\
\hline$K R R 1$ & 0.19 & $1.2 \mathrm{E}-5$ \\
\hline RANBP6 & 0.14 & $1.7 \mathrm{E}-5$ \\
\hline$B R I X 1$ & 0.04 & $2.4 \mathrm{E}-5$ \\
\hline TM2D3 & 0.21 & $2.9 \mathrm{E}-5$ \\
\hline$S C P 2$ & 0.30 & $3.3 \mathrm{E}-5$ \\
\hline METTL18 & 0.31 & $6.2 \mathrm{E}-5$ \\
\hline$C C D C 121$ & 0.17 & $6.4 \mathrm{E}-5$ \\
\hline SRP68 & 0.31 & $6.5 \mathrm{E}-5$ \\
\hline CHTF8 & 0.20 & $6.6 \mathrm{E}-5$ \\
\hline TRIM21 & 0.20 & $7.1 \mathrm{E}-5$ \\
\hline
\end{tabular}

b

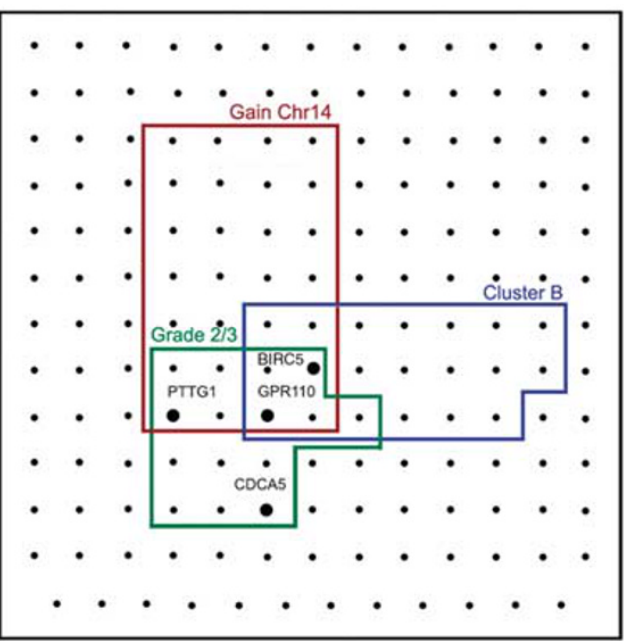

C
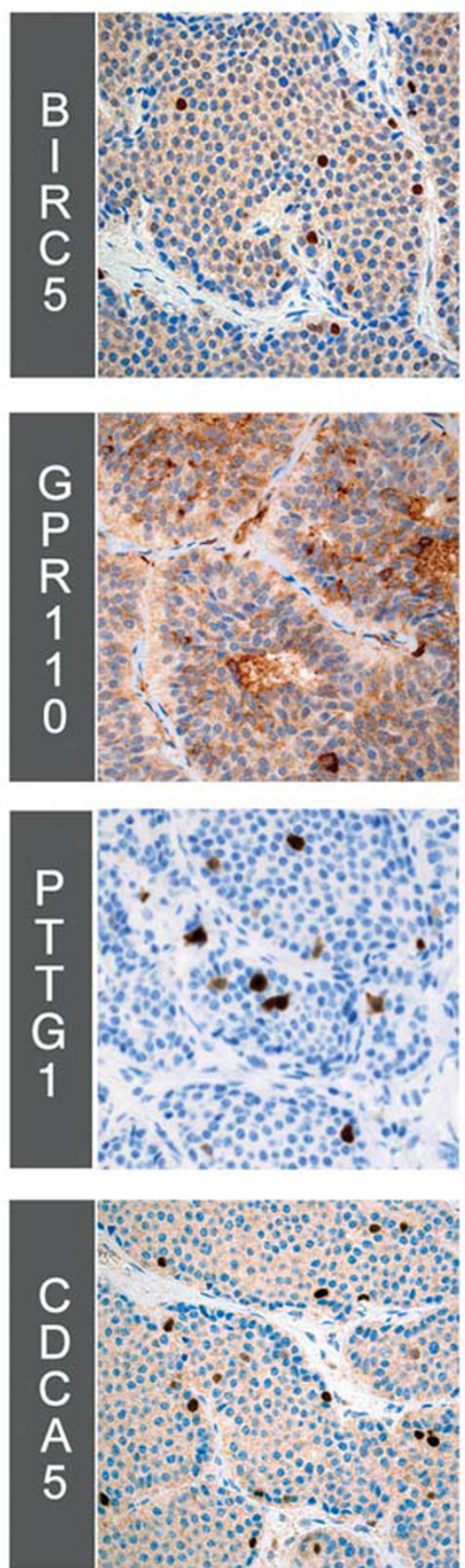
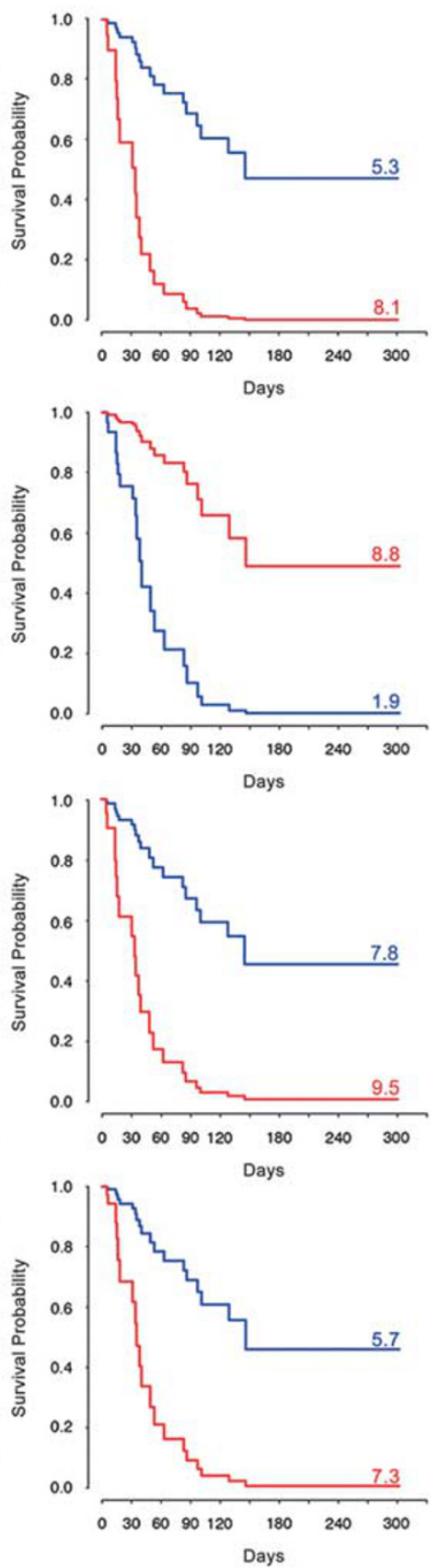

Figure 5 For caption see page 626 
pathways, for example, activation of NUPR1, which were not involved in the regulation of cell proliferation. The relatively small number of tumors in cluster $\mathrm{C}$ precludes a definitive characterization of this cluster.

Grading of neuroendocrine tumors provides information for prognostication and patient management. ${ }^{8,9}$ The current WHO grading system is based on an assessment of tumor cell proliferation, and classifies the majority of small intestinal neuroendocrine tumors as low proliferation neoplasms (grade 1). ${ }^{5,33}$ In order to identify the molecular alterations associated with tumor grade, we compared low-grade tumors to intermediate- and highgrade tumors and identified a small but distinct set of differentially expressed genes. Pathway analysis indicated activation of cell cycle regulators such as E2Fs, TBX1 and FOXM1 in intermediate-grade

Table 3 List of therapeutic drug targets in small intestinal neuroendocrine tumors identified by gene expression profiling

\begin{tabular}{|c|c|c|c|c|c|c|}
\hline \multirow[b]{2}{*}{ Target } & \multirow[b]{2}{*}{ Type of protein } & \multirow[b]{2}{*}{ Drug } & \multicolumn{2}{|c|}{ SI-NETS } & \multicolumn{2}{|c|}{ GOT1 } \\
\hline & & & $\operatorname{LogFC}$ & $\begin{array}{c}\text { Adj. } \\
\text { P-value }\end{array}$ & $\operatorname{LogFC}$ & $\begin{array}{c}\text { Adj. } \\
\text { P-value }\end{array}$ \\
\hline RET & Kinase & $\begin{array}{l}\text { Sorafenib*, sunitinib*, regorafenib, } \\
\text { vandetanib, cabozantinib }\end{array}$ & 4.61 & $3.5 \mathrm{E}-08$ & 7.11 & $1.2 \mathrm{E}-09$ \\
\hline SSTR2 & GPC receptor & Pasireotide $^{*}$, lanreotide ${ }^{*}$, octreotide* & 3.85 & 8.7E-11 & 4.32 & $7.3 \mathrm{E}-07$ \\
\hline AKT3 & Kinase & MK-2206, perifosine & 3.62 & $3.1 \mathrm{E}-15$ & 2.51 & $1.2 \mathrm{E}-04$ \\
\hline TUBB8 & Other & Cabazitaxel, epothilone & 3.29 & $7.5 \mathrm{E}-09$ & 2.75 & $2.1 \mathrm{E}-03$ \\
\hline FGFR1 & Kinase & Regorafenib, nintedanib, dovitinib, BGJ398 & 2.52 & $2.5 \mathrm{E}-10$ & 3.91 & $2.4 \mathrm{E}-05$ \\
\hline HDAC5 & Transcription regulator & Vorinostat, belinostat & 2.50 & $7.4 \mathrm{E}-11$ & 3.19 & $4.1 \mathrm{E}-06$ \\
\hline RARG & Nuclear receptor & Tretinoin, isotretinoin, fenretinide & 2.01 & $1.5 \mathrm{E}-09$ & 0.78 & $4.0 \mathrm{E}-01$ \\
\hline PDGFRB & Kinase & $\begin{array}{l}\text { Imatinib, sorafenib*, sunitinib*, dasatinib, } \\
\text { pazopanib*, regorafenib, }^{*}\end{array}$ & 2.01 & $1.8 \mathrm{E}-05$ & -7.58 & $7.3 \mathrm{E}-07$ \\
\hline PARP1 & Enzyme & Veliparib, olaparib, rucaparib & 1.99 & $2.2 \mathrm{E}-05$ & 1.20 & $1.1 \mathrm{E}-01$ \\
\hline FLT1/VEGFR1 & Kinase & $\begin{array}{l}\text { Sunitinib*, pazopanib, axitinib, } \\
\text { regorafenib, motesanib }\end{array}$ & 1.96 & $2.4 \mathrm{E}-05$ & -2.11 & $2.0 \mathrm{E}-02$ \\
\hline FGFR3 & Kinase & Nintedanib, dovitinib, BGJ398 & 1.82 & $2.5 \mathrm{E}-05$ & -6.28 & $9.3 \mathrm{E}-08$ \\
\hline HSP90AA1 & Enzyme & $\begin{array}{l}\text { Retaspimycin, tanespimycin, ganetespib, } \\
\text { alvespimycin, }\end{array}$ & 1.79 & $1.5 \mathrm{E}-07$ & 2.05 & $2.6 \mathrm{E}-04$ \\
\hline VEGFB & Growth factor & Bevacizumab*, aflibercept & 1.77 & $1.6 \mathrm{E}-12$ & 2.88 & $8.1 \mathrm{E}-06$ \\
\hline CDK9 & Kinase & Alvocidib, P276-00 & 1.68 & $9.2 \mathrm{E}-11$ & 1.28 & $5.5 \mathrm{E}-03$ \\
\hline HSP90AB1 & Enzyme & $\begin{array}{l}\text { Retaspimycin, tanespimycin, ganetespib, } \\
\text { alvespimycin, }\end{array}$ & 1.59 & $4.6 \mathrm{E}-08$ & 3.41 & $2.8 \mathrm{E}-05$ \\
\hline CDK4 & Kinase & P276-00, LEE011, palbociclib & 1.16 & $1.0 \mathrm{E}-07$ & 3.21 & $8.6 \mathrm{E}-05$ \\
\hline
\end{tabular}

For each target, corresponding anticancer drugs are listed. Anticancer drugs evaluated in patients with gastroenteropancreatic neuroendocrine tumors are indicated by an asterisk. Candidate targets are listed according to decreasing gene expression values (logFC). SI-NETs, small intestinal neuroendocrine tumors.

Figure 5 Gene expression associated with patient survival. (a) Survival-related genes in small intestinal neuroendocrine tumors represented multiple cellular functions, including cell cycle progression (CDCA5, CENPF and CHTF8), apoptosis (FAM9C and ARHGAP11A), DNA damage response (CDCA5 and GTSE1), invasion (GTSE1, ADAMTS12 and ARHGAP11A), and immune response (TRIM40, ADAMTS12 and TRIM21). The 20 genes most associated with shorter and longer patient survival are listed (Cox proportional hazards model, adjusted $P$-value $<0.12$ ). Asterisks indicate proteins localized to tumor tissues by immunohistochemistry. (b) Venn diagram illustrating the overlap of survival-related genes with differentially expressed genes in tumors belonging to cluster B, of grade $2 / 3$ and with gain of chromosome 14. One-third of survival-related genes (49 of 168) were represented in one or several of the subgroups. The majority of survival-related genes were not represented in any of the subgroups, indicating that a large proportion of gene expression affecting patient survival was not related to tumor cluster, grade, or chromosomal gain. The Venn diagram was generated by comparing survival-related genes (adjusted $P$-value $\leq 0.12, n=168$, each gene represented by a dot) with differentially expressed genes in cluster B vs A (adjusted $P$-value $<0.005, n=3112$ ), grade $2 / 3$ vs grade 1 (adjusted $P$-value $<0.05, n=70$ ), and gain of chromosome 14 vs no gain of chromosome 14 (adjusted $P$-value $<0.05, n=181$ ). (c) Examples of survival-related genes in small intestinal neuroendocrine tumors. The survival probabilities according to the Cox regression model have been plotted for each gene, and corresponding mRNA abundance values are shown. The red and blue curves refer to patients with high and low expression (defined as the first and third quartile in the data set; expression values are indicated at the curve). Corresponding proteins were localized to tumor cells by immunohistochemistry. High expression of BIRC5 (survivin), a promoter of chromosome alignment and separation, and inhibitor of apoptosis, was associated with shorter patient survival (Exp Coef 2.2, $P$-value $<0.0003$ ). BIRC5 was significantly upregulated in tumors of cluster B, grade $2 / 3$ tumors and tumors with gain of chromosome 14. High expression of GPR110, a G protein-coupled receptor of unknown function, was associated with longer patient survival (Exp Coef $0.3, P$-value $<0.0002$ ). GPR110 was significantly downregulated in tumors of cluster B, those of grade 2/3 and those with gain of chromosome 14. High expression of PTTG1 (securin), a transforming protein regulating chromosome stability and inhibiting P53 and apoptosis, was associated with shorter patient survival (Exp Coef 3.1, $P$-value $<0.0002$ ). PTTG1 was significantly upregulated in tumors of grade $2 / 3$ and those with gain of chromosome 14 . High expression of CDCA5 (sororin), a regulator of sister chromatid cohesion and separation, was associated with shorter patient survival (Exp Coef 3.3, P-value $<0.00004$ ). CDCA5 was significantly upregulated in tumors of grade $2 / 3$. 
Sorafenib

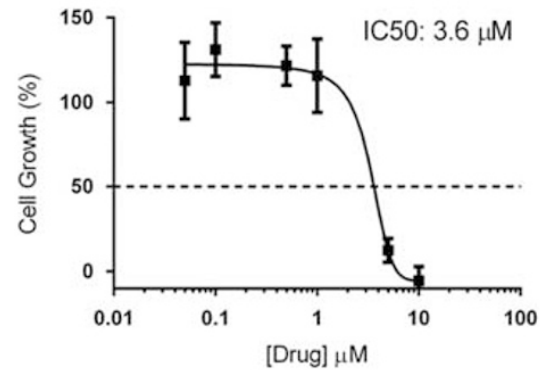

Cabozantinib

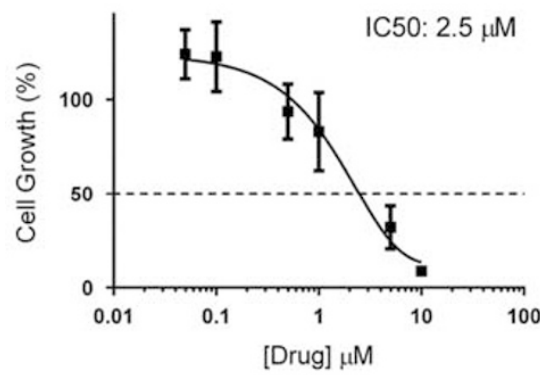

Veliparib

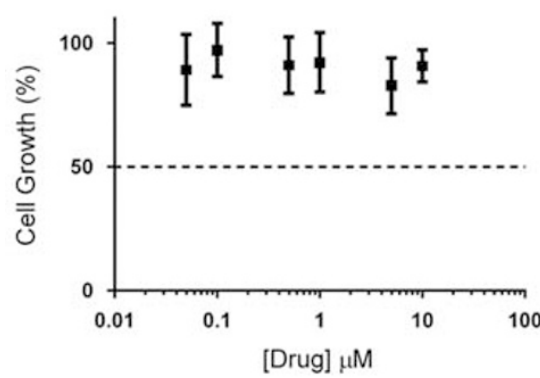

Sunitinib

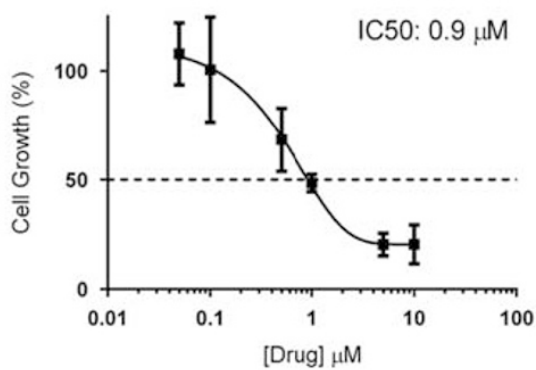

MK-2206

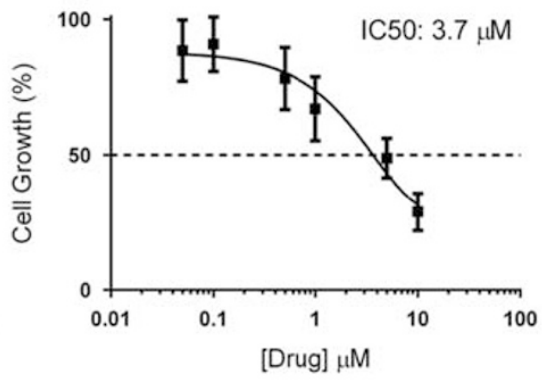

Alvespimycin

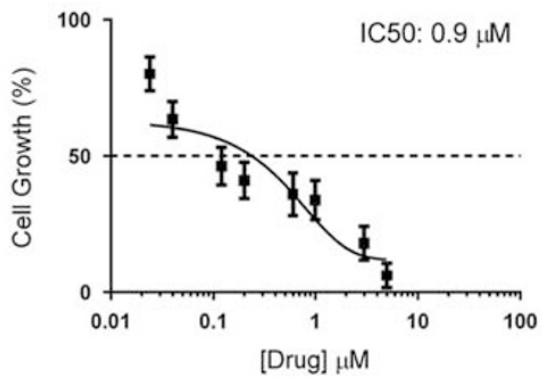

Regorafenib

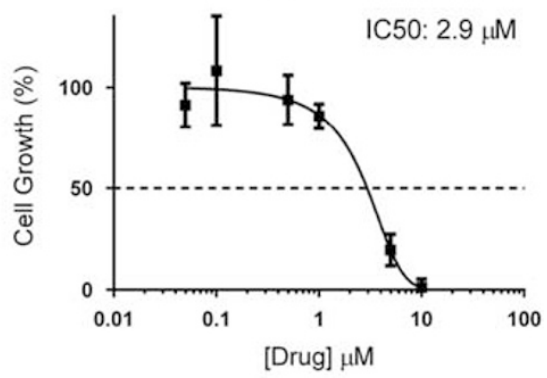

Vorinostat

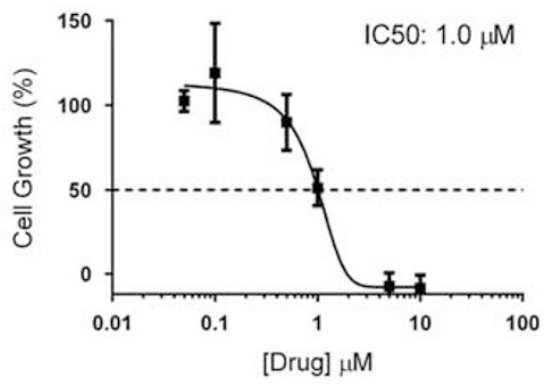

P276-00

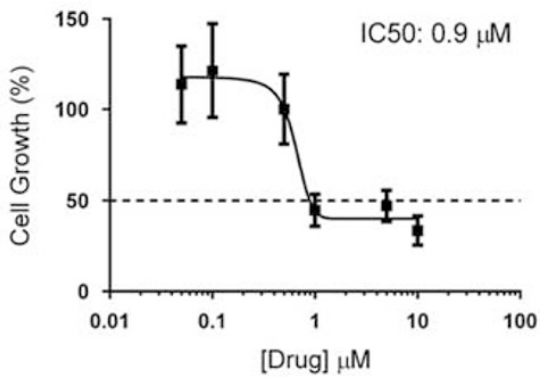

Figure 6 Predicted anticancer drugs inhibit the growth of neuroendocrine tumor cells. Multi-tyrosine kinase inhibitors sorafenib, sunitinib, regorafenib and cabozantinib effectively inhibit GOT1 cell growth with IC $_{50}$ values in the micromolar range. Inhibitors of AKT (MK-2206), HDAC (vorinostat), HSP90 (alvespimycin) and CDK4/9 (P276-00) also inhibited GOT1 cell growth at micromolar concentrations, whereas inhibition of PARP1 (veliparib) had no effect. GOT1 cells were treated with anticancer drugs at various concentrations for 4 days. Cell viability was estimated using AlamarBlue ${ }^{\circledR}$. Data points are the mean values of three individual experiments carried out in triplicate $(n=9)$. Fitting of curves was done in GraphPad Prism software v6.04 using log (inhibitor) vs response nonlinear fit with variable slope. $\mathrm{IC}_{50}$ was interpolated at $\mathrm{Y}=50$ and bars denote \pm s.d.

tumors, but no alterations directly involving p53. The most significantly activated regulator, however, was PTGER2, which promotes tumor cell proliferation as well as invasion and angiogenesis. ${ }^{34}$ This finding was unexpected, and indicates that immune mechanisms are activated in tumors of higher grade. The observation may be exploited clinically, as COX2 inhibitors and selective PTGER2 inhibitors are available for targeting of PTGER2 in tumors. ${ }^{34}$

Small intestinal neuroendocrine tumors are characterized by recurrent chromosomal alterations, the most frequent involving loss of chromosome 18. Chromosomal gains involving chromosomes 4, 5, 7, 14 and 20 are observed in a small proportion of tumors and have been associated with shorter survival. ${ }^{20,35}$ In order to further characterize tumors with gain of chromosome 14, we analyzed differentially expressed genes in this group and found FOXM1 to be the most significantly activated upstream regulator. FOXM1 is an oncogenic transcription factor promoting cell cycle progression and resistance to genotoxic drugs. ${ }^{36,37}$ Recently, a strong association between FOXM1 and metastases was reported for gastroenteropancreatic neuroendocrine tumors. $^{38}$ Therapies targeting FOXM1 are under way, and may be considered in the future. ${ }^{39}$

The clinical course of patients with metastatic disease is difficult to predict. In this study, we identified a series of genes that were associated with patient survival, two of which (BIRC5 and CDKN1B) have previously been described in small intestinal neuroendocrine tumors. ${ }^{27-29}$ The current WHO grading system for neuroendocrine tumors, which is used for prognostication, is based solely on an 
estimation of tumor cell proliferation. ${ }^{5}$ Our results indicate that prognostication could be further developed to include biomarkers that reflect other properties of tumor cells, including tumor-host interactions.

We searched expression profiles for novel therapeutic targets by comparing tumors with normal small intestinal mucosa. We chose intestinal mucosa as control because it represents the primary site of tumors. However, intestinal mucosa may not be optimal as control tissue because its content of endocrine cells is low, and the results from such a comparison must therefore be interpreted with caution. Based on this comparison, we were able to identify a number of candidate targets with corresponding anticancer drugs currently in clinical trials. Somatostatin receptor 2 (SSTR2), which can be targeted by long-acting somatostatin analogs, was found to be expressed in all tumors but had highest expression in tumors of cluster A. The efficacy of long-acting somatostatin analogs or radiolabeled analogs $7,40,41$ may therefore be influenced by cluster designation. However, multiple factors, including receptor profile, tumor heterogeneity, affinity of analogs and choice of radionuclide, also influence the efficacy of somatostatin receptor targeted therapy and the relative importance of cluster designation remains to be determined. Components of proliferative pathways such as growth factors (VEGFB) and receptor tyrosine kinases (RET, FGFR1/3, PDGFRB and FLT1) were frequently identified as drug targets, and corresponding tyrosine kinase inhibitors were shown to effectively inhibit the growth of neuroendocrine tumor cells in vitro. Thus, receptor tyrosine kinases represent key signaling pathways, which control sustained tumor cell proliferation, ${ }^{42}$ and targeting of these pathways appears to be a promising strategy in patients with metastatic disease. Inhibitors of transcriptional regulators (HDAC), molecular chaperones (HSP90) and signal transduction molecules (AKT3) were also found to effectively inhibit the growth of neuroendocrine tumor cells, but they have not yet been evaluated in clinical trials. These findings suggest that treatment with these inhibitors should be further evaluated.

\section{Acknowledgments}

We are grateful to Gülay Altiparmak, Linda Inge and Pauline Brattberg for their expert technical assistance. We thank Jonas Nilsson for critically reviewing the manuscript. This study was supported by the Swedish Cancer Society (to O Nilsson), the Sahlgrenska Academy (ALF agreement) (to O Nilsson and B Wängberg), the Assar Gabrielsson Research Foundation and Sahlgrenska University Hospital Research Funds (to E Andersson), the Life Science Area of Advance at Chalmers University of Technology (to E Kristiansson) and the Swedish Research Council and the Wallenberg Foundation (to E Kristiansson).

\section{Disclosure/conflict of interest}

The authors declare no conflict of interest.

\section{References}

1 Yao JC, Hassan M, Phan A et al. One hundred years after 'carcinoid': epidemiology of and prognostic factors for neuroendocrine tumors in 35,825 cases in the United States. J Clin Oncol 2008;26:3063-3072.

2 Møller JE, Pellikka PA, Bernheim AM et al. Prognosis of carcinoid heart disease: analysis of 200 cases over two decades. Circulation 2005;112:3320-3327.

3 Norlén O, Stålberg P, Öberg K et al. Long-term results of surgery for small intestinal neuroendocrine tumors at a tertiary referral center. World J Surg 2012;36: 1419-1431.

4 Strosberg JR, Weber JM, Feldman M et al. Prognostic validity of the American Joint Committee on Cancer staging classification for midgut neuroendocrine tumors. J Clin Oncol 2013;31: 420-425.

5 Capella C, Arnold R, Klimstra DS et al. Neuroendocrine neoplasms of the small intestine. In: Bosman TF, Carneiro F, Rhuban RH (eds). WHO Classification of Tumours fo the Digestive System, 4th edn. IARC: Lyon, France, 2010, pp 102-107.

6 Modlin IM, Moss SF, Chung DC et al. Priorities for improving the management of gastroenteropancreatic neuroendocrine tumors. J Natl Cancer Inst 2008;100: 1282-1289.

7 Rinke A, Müller HH, Schade-Brittinger C et al. Placebocontrolled, double-blind, prospective, randomized study on the effect of octreotide LAR in the control of tumor growth in patients with metastatic neuroendocrine midgut tumors: a report from the PROMID Study Group. J Clin Oncol 2009;27:4656-4663.

8 Boudreaux JP, Klimstra DS, Hassan MM et al. The NANETS consensus guideline for the diagnosis and management of neuroendocrine tumors: welldifferentiated neuroendocrine tumors of the Jejunum, Ileum, Appendix, and Cecum. Pancreas 2010;39: 753-766.

9 Pape UF, Perren A, Niederle B et al. ENETS Consensus Guidelines for the management of patients with neuroendocrine neoplasms from the jejuno-ileum and the appendix including goblet cell carcinomas. Neuroendocrinology 2012;95:135-156.

10 Jann H, Roll S, Couvelard A et al. Neuroendocrine tumors of midgut and hindgut origin: tumor-nodemetastasis classification determines clinical outcome. Cancer 2011;117:3332-3341.

11 Modlin IM, Champaneria MC, Chan AK et al. A threedecade analysis of 3,911 small intestinal neuroendocrine tumors: the rapid pace of no progress. Am J Gastroenterol 2007;102:1464-1473.

12 Lawrence B, Gustafsson BI, Chan A et al. The epidemiology of gastroenteropancreatic neuroendocrine tumors. Endocrinol Metab Clin North Am 2011;40:1-18 vii.

13 Macconaill LE, Garraway LA. Clinical implications of the cancer genome. J Clin Oncol 2010;28:5219-5228.

14 McDermott U, Settleman J. Personalized cancer therapy with selective kinase inhibitors: an emerging paradigm in medical oncology. J Clin Oncol 2009;27:5650-5659. 
15 Garnett MJ, Edelman EJ, Heidorn SJ et al. Systematic identification of genomic markers of drug sensitivity in cancer cells. Nature 2012;483:570-575.

16 Banck MS, Kanwar R, Kulkarni AA et al. The genomic landscape of small intestine neuroendocrine tumors. J Clin Invest 2013;123:2502-2508.

17 Francis JM, Kiezun A, Ramos AH et al. Somatic mutation of CDKN1B in small intestine neuroendocrine tumors. Nat Genet 2013;45:1483-1486.

18 Sorlie T, Perou CM, Tibshirani R et al. Gene expression patterns of breast carcinomas distinguish tumor subclasses with clinical implications. Proc Natl Acad Sci USA 2001;98:10869-10874.

19 Norum JH, Andersen K, Sorlie T. Lessons learned from the intrinsic subtypes of breast cancer in the quest for precision therapy. Br J Surg 2014;101:925-938.

20 Andersson E, Swärd C, Stenman G et al. Highresolution genomic profiling reveals gain of chromosome 14 as a predictor of poor outcome in ileal carcinoids. Endocr Relat Cancer 2009;16:953-966.

21 Smyth GK. Linear models and empirical bayes methods for assessing differential expression in microarray experiments. Stat Appl Genet Mol Biol 2004;3: Article 3.

22 Bolstad BM, Irizarry RA, Åstrand M et al. A comparison of normalization methods for high density oligonucleotide array data based on variance and bias. Bioinformatics 2003;19:185-193.

23 Ritchie ME, Silver J, Oshlack A et al. A comparison of background correction methods for two-colour microarrays. Bioinformatics 2007;23:2700-2707.

24 Benjamini YH, Y. Controlling the false discovery rate: a practical and powerful approach to multiple testing. J R Stat Soc Ser B (Stat Method) 1995;57:289-300.

25 Kramer A, Green J, Pollard J Jr et al. Causal analysis approaches in ingenuity pathway analysis. Bioinformatics 2014;30:523-530.

26 Kölby L, Bernhardt P, Ahlman H et al. A transplantable human carcinoid as model for somatostatin receptor-mediated and amine transporter-mediated radionuclide uptake. Am J Pathol 2001;158:745-755.

27 Grabowski P, Griss S, Arnold CN et al. Nuclear survivin is a powerful novel prognostic marker in gastroenteropancreatic neuroendocrine tumor disease. Neuroendocrinology 2005;81:1-9.

28 Grabowski P, Schrader J, Wagner J et al. Loss of nuclear p27 expression and its prognostic role in relation to cyclin E and p53 mutation in gastroenteropancreatic neuroendocrine tumors. Clin Cancer Res 2008;14: 7378-7384.

29 Kim HS, Lee HS, Nam KH et al. p27 loss is associated with poor prognosis in gastroenteropancreatic neuro endocrine tumors. Cancer Res Treat 2014;46:383-392.

30 Massague J. TGFbeta in cancer. Cell 2008;134:215-230.

31 Massague J. TGFbeta signalling in context. Nat Rev Mol Cell Biol 2012;13:616-630.

32 Hamidi T, Cano CE, Grasso D et al. Nupr1-aurora kinase A pathway provides protection against metabolic stress-mediated autophagic-associated cell death. Clin Cancer Res 2012;18:5234-5246.

33 Cunningham JL, Grimelius L, Sundin A et al. Malignant ileocaecal serotonin-producing carcinoid tumours: the presence of a solid growth pattern and/or Ki67 index above $1 \%$ identifies patients with a poorer prognosis. Acta Oncol 2007;46:747-756.

34 Jiang J, Dingledine R. Role of prostaglandin receptor EP2 in the regulations of cancer cell proliferation, invasion, and inflammation. J Pharmacol Exp Ther 2013;344:360-367.

35 Hashemi J, Fotouhi O, Sulaiman L et al. Copy number alterations in small intestinal neuroendocrine tumors determined by array comparative genomic hybridization. BMC Cancer 2013;13:505.

36 Halasi M, Gartel AL. FOX(M1) news-it is cancer. Mol Cancer Ther 2013;12:245-254.

37 Zona S, Bella L, Burton MJ et al. FOXM1: an emerging master regulator of DNA damage response and genotoxic agent resistance. Biochim Biophys Acta 2014;1839:1316-1322.

38 Briest F, Berg E, Grass I et al. FOXM1: a novel drug target in gastroenteropancreatic neuroendocrine tumors. Oncotarget 2015;6:8185-8199.

39 Halasi M, Gartel AL. Targeting FOXM1 in cancer. Biochem Pharmacol 2013;85:644-652.

40 Kwekkeboom DJ, de Herder WW, Kam BL et al. Treatment with the radiolabeled somatostatin analog [177 Lu-DOTA 0,Tyr3] octreotate: toxicity, efficacy, and survival. J Clin Oncol 2008;26:2124-2130.

41 Vinjamuri S, Gilbert TM, Banks $\mathrm{M}$ et al. Peptide receptor radionuclide therapy with (90)Y-DOTATATE/(90)Y-DOTATOC in patients with progressive metastatic neuroendocrine tumours: assessment of response, survival and toxicity. Br J Cancer 2013;108: 1440-1448.

42 Cunningham JL, Janson ET. The biological hallmarks of ileal carcinoids. Eur J Clin Invest 2011;41:1353-1360.

Supplementary Information accompanies the paper on Modern Pathology website (http://www.nature.com/ modpathol) 